\title{
Trophic resource partitioning drives fine-scale coexistence in cryptic bat species
}

\author{
Roberto Novella-Fernandez $^{1}$ | Carlos Ibañez ${ }^{2}$ | Javier Juste ${ }^{2,3}$ | Elizabeth L. Clare ${ }^{4}$ | \\ C. Patrick Doncaster ${ }^{1}$ (D) | Orly Razgour ${ }^{1,5}$ (D)
}

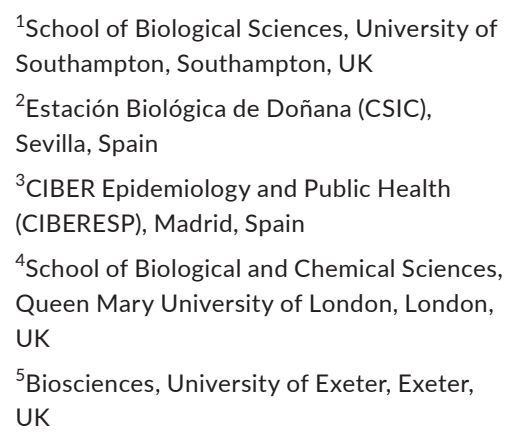

\begin{abstract}
Understanding the processes that enable species coexistence has important implications for assessing how ecological systems will respond to global change. Morphology and functional similarity increase the potential for competition, and therefore, cooccurring morphologically similar but genetically unique species are a good model system for testing coexistence mechanisms. We used DNA metabarcoding and highthroughput sequencing to characterize for the first time the trophic ecology of two recently described cryptic bat species with parapatric ranges, Myotis escalerai and Myotis crypticus. We collected fecal samples from allopatric and sympatric regions and from syntopic and allotopic locations within the sympatric region to describe the diets both taxonomically and functionally and compare prey consumption with prey availability. The two bat species had highly similar diets characterized by high arthropod diversity, particularly Lepidoptera, Diptera and Araneae, and a high proportion of prey that is not volant at night, which points to extensive use of gleaning. Diet overlap at the prey item level was lower in syntopic populations, supporting trophic shift under fine-scale co-occurrence. Furthermore, the diet of M. escalerai had a marginally lower proportion of not nocturnally volant prey in syntopic populations, suggesting that the shift in diet may be driven by a change in foraging mode. Our findings suggest that fine-scale coexistence mechanisms can have implications for maintaining broad-scale diversity patterns. This study highlights the importance of including both allopatric and sympatric populations and choosing meaningful spatial scales for detecting ecological patterns. We conclude that a combination of high taxonomic resolution with a functional approach helps identify patterns of niche shift.
\end{abstract}

\section{KEYWORDS}

bats, cryptic species, DNA metabarcoding, interspecific competition, molecular diet analysis, Myotis nattereri species complex, species coexistence, trophic partitioning 


\section{1 | INTRODUCTION}

Understanding the processes that enable species coexistence is a key theme of ecology with important implications for interpreting diversity patterns and predicting how systems respond to global change (Valladares et al., 2015). Interspecific competition is thought to have a major influence on community structure for many taxonomic groups (Weiher \& Keddy, 1999). Niche theory (Chase \& Leibold, 2003; Chesson, 2000; Letten et al., 2017) asserts that species coexistence is promoted through differential use of resources driven by functional differences between species, which results in communities that tend to be assembled by functionally dissimilar species (Schoener, 1974). This has been shown in numerous cases, including fish (Ross, 1986), shorebirds (Bocher et al., 2014), and rodent communities (Codron et al., 2015). Alternatively, community structure and coexistence, primarily in sessile organisms, have been often explained through neutral processes, such as dispersal or stochasticity (The neutral theory of biodiversity and biogeography; Hubbell, 2001). This framework has been often used as a null model to evaluate whether observed patterns deviate from neutral expectations (Alonso et al., 2006; McGill et al., 2006). Yet, some studies of mobile organisms have failed to identify evidence of resource partitioning (e.g., Luiselli, 2008), suggesting that in some cases biotic interactions only play a minor role in governing community assembly, perhaps because resources are not limiting (Salinas-Ramos et al., 2020), and therefore, neutral processes likely play a more important role.

Morphologically similar species pose a challenge for understanding mechanisms of coexistence from a niche theory perspective because they are more likely to be functionally similar, and therefore less likely to be able to use resources in a different way, a prerequisite for resource partitioning (Weiher \& Keddy, 1999). Consequently, considerable attention has been given to understanding resource partitioning among morphologically identical (cryptic) or similar co-occurring species (e.g., Gabaldón et al., 2013; Jiang et al., 2008; Razgour et al., 2011). Many studies have focused on the trophic dimension, an important aspect of species' ecological niche (Schoener, 1974). DNA metabarcoding and high-throughput sequencing (molecular diet analysis) approaches helped overcome many of the limitations of traditional morphological methods (Sousa et al., 2019), opening the door to new opportunities for studying mechanisms of species coexistence (Arrizabalaga-Escudero et al., 2018; Krüger et al., 2014;
Razgour et al., 2011). However, the majority of coexistence studies focus on only sympatric populations, preventing an evaluation of how the presence of a competitor may change resource use, thus limiting the power of inferences (Salinas-Ramos et al., 2020). Moreover, most studies also focus on diet only, and disregard prey selection relative to prey availability or resource limitation (Salinas-Ramos et al., 2020). Accounting for prey selection (e.g., Rytkönen et al., 2019) can provide a more complete picture of consumer trophic preferences (Lawlor, 1980).

The processes that govern community assembly, including coexistence mechanisms, vary with spatial scale (Lewis et al., 2015; Snyder \& Chesson, 2004; Viana \& Chase, 2019), yet spatial scale is rarely considered in coexistence studies (Hart et al., 2017). A better understanding of the scale of coexistence mechanisms and how different processes interact is important for both basic and applied ecology (Peixoto et al., 2018).

This study aims to identify whether trophic ecology enables morphologically similar species to coexist across spatial scales. We focus on two recently described insectivorous bat species whose trophic ecology has not been studied to date, Myotis crypticus and Myotis escalerai (Figure 1). Both species forage in forests and are morphologically nearly identical, only distinguishable by a small difference in the uropatagium and its terminal row of hairs (Juste et al., 2019). These bats are restricted to the Western Mediterranean Basin, where they overlap across the north of the Iberian Peninsula, but at the fine-scale are known to co-occur only in a few locations (Juste et al., 2019). Phylogeographic analysis and species distribution modeling suggest that their ranges have been shaped by competition (Razgour et al., 2015). These bats therefore provide an excellent case study for understanding mechanisms of coexistence among morphologically similar species. We use DNA metabarcoding and high-throughput sequencing to characterize the trophic ecology of M. crypticus and M. escalerai by analyzing their taxonomic and functional diets and their prey selection relative to prey availability in sympatry versus allopatry at both fine and regional spatial scales. Given their near identical morphology and echolocation calls, the overall trophic niches of the two bats are expected to be similar and niche overlap should be high. We hypothesize that if resource partitioning is the main process facilitating coexistence: (a) trophic niche overlap and diet similarity will be higher in allopatry than sympatry (e.g., Klawinski et al., 1994), and (b) differences in trophic niche overlap will be most pronounced at the fine spatial scale where individuals of the two species directly co-occur.
FIGURE 1 Myotis crypticus(a) andMyotis escalerai(b) in Spain. Photographs taken by Roberto NovellaFernandez and Daniel Fernandez-Alonso
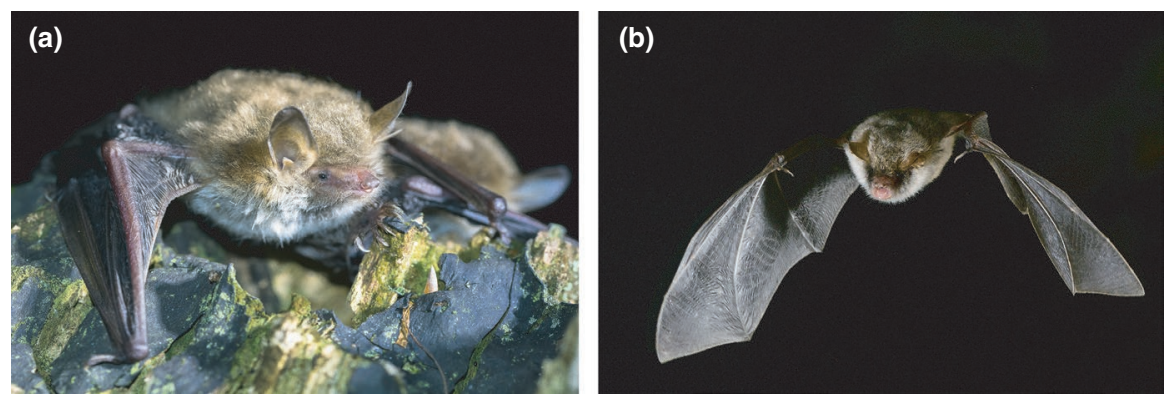


\section{2 | METHODS}

\section{1 | Sampling design}

Sampling took place in the Iberian Peninsula, focusing on two sympatric regions in the north where both Myotis escalerai and Myotis crypticus are found (La Rioja-Soria and southern Cantabria), and two allopatric regions: the south (Andalusia: Jaen and Granada), where only M. escalerai is found, and the north Atlantic coast (northern Cantabria), where only M. crypticus is found. Additionally, we sampled a single swarming site in Catalonia, where the two species use during the autumn mating season (Figure 2). Within each region, 9-24 locations were sampled based on suitable habitat and accessibility, using monofilament mist nets and a harp trap placed over water sources, forest paths and cave entrances. The sampling period extended from June to September 2017, for a total of 68 sampling nights (Table S1 for list of sampling locations). Captured bats were kept in individual cotton bags for up to $1 \mathrm{hr}$. We collected fecal samples from the cotton bags for diet analysis, and biopsy punches $(3 \mathrm{~mm})$ from the wing membrane of the bats to confirm species identification. Dropping samples and wing biopsies were stored for each bat individually in absolute and $70 \%$ ethanol, respectively. Bat sampling was carried out under local permits and ethical approval from the University of Southampton (study ID: 26627).

We sampled the arthropod community in bat sampling locations using vegetation sweeping (Barclay, 1991; Swift \& Racey, 2002) to assess bat prey selectivity relative to prey availability (Jones, 1990; Kunz, 2009). We chose vegetation sweeping because of the expected low flight and gleaning behavior of the species based on their morphology and echolocation calls, and the foraging behavior of the morphologically similar better studied member of the cryptic species complex, Myotis nattereri (de Jong, 1995). During bat sampling nights, we set linear transects in each habitat type in the sampling location and swept the vegetation along each transect. After 10 sweeps, we collected the capture into a plastic bag and moved five steps further without sampling to increase spatial representativeness. Each sampling unit of 10 sweeps and five steps forward was repeated 5-10 times until the capture size was considered representative ( $>100$ individuals). Transect length ranged between 30 and $80 \mathrm{~m}$. Arthropod specimens captured were separated from vegetation remains in the field and stored in $70 \%$ ethanol. A total of 43 sweeping samples from 23 locations with at least three bat individuals were selected (Table S1).

\subsection{DNA extractions and species confirmation}

Bat species identity was confirmed in the Estación Biológica de Doñana Laboratory of Molecular Ecology (LEM, EBD-CSIC, Spain). DNA was extracted from wing biopsy punches through precipitation with isopropanol. Part of the hypervariable region of the mIDNA control region was amplified using the primers CSBC-F 5'-CCTCTTAAATAAGACATCTCGATGG-3' (Wilkinson \& Chapman, 1992) and HV2-Mna-R 5'-ATGCGTGCGTGTGTAATGTC-3' (GarciaMudarra et al., In Press). Species specific differential amplification patterns for this primer set were used to confirm species identity through gel electrophoresis (Garcia-Mudarra et al., In Press).

DNA was extracted from all bat dropping samples using the Qiagen DNA stool mini kit, following the protocol in Zeale et al. (2011). From the 43 selected sweeping samples, all arthropod individuals $(N=8,366)$ were first identified morphologically to taxonomic order. Subsequently, whole specimens, if smaller than a drosophila, or a specimen part (leg or head) if larger, were separated out, dried and pooled together for DNA extraction. Arthropod DNA was

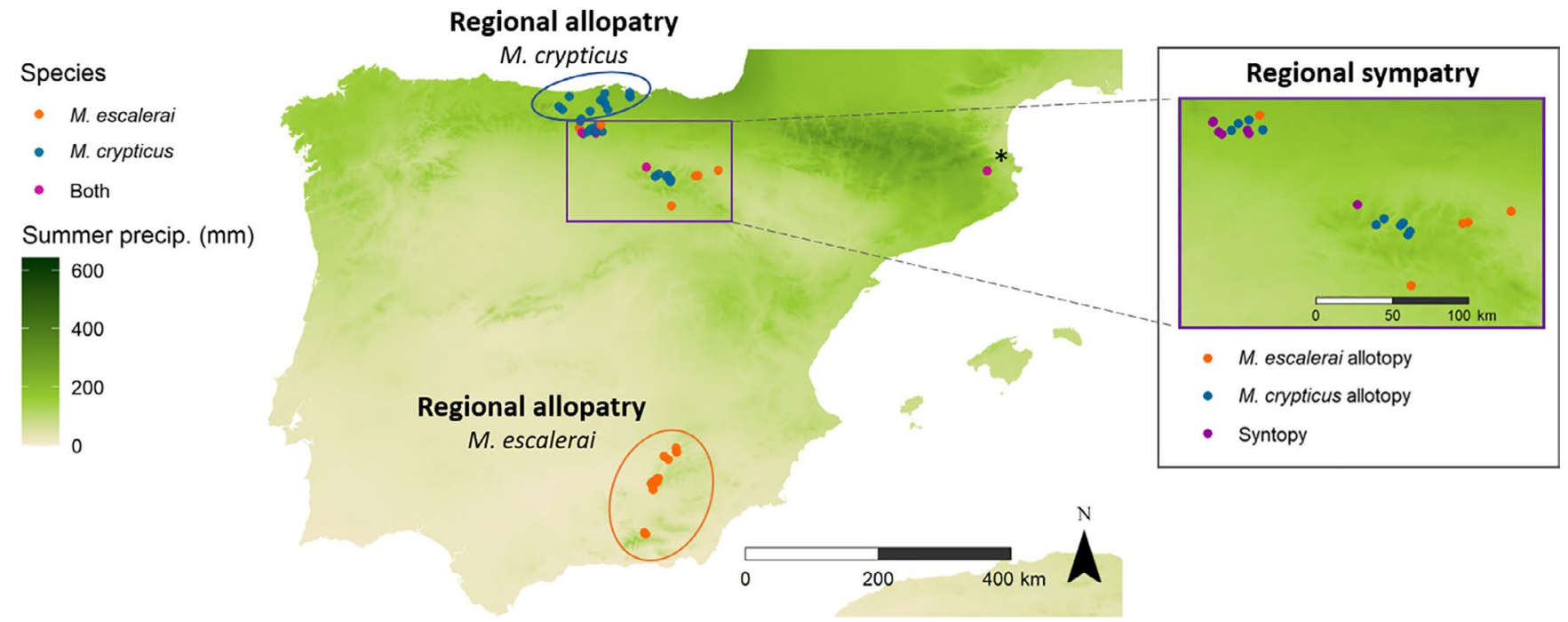

FIGURE 2 Sampling locations overlaying summer precipitation across Spain, with gradient from dry Mediterranean to wet Atlantic. Ovals encompass the two allopatric regions (Granada and Jaen forM. escaleraiand northern Cantabria forM. crypticus). The rectangle encompasses the sympatric region (La Rioja and the Mediterranean climatic zone at the south of Cantabria). Insert shows the sympatric region with syntopic (red) versus allotopic (blue and orange) locations. Black star near the sympatric location at the eastern side of the main map in Catalonia denotes a swarming site and was excluded from the analysis 
extracted using the NucleoSpin DNA Insect kit with up to $35 \mathrm{mg}$ of sample dry weight in each tube. Larger samples were split into several tubes. The following modifications were applied to the kit extraction protocol: In steep 2, vortex for $20 \mathrm{~min}$ in the MN Bead Tube Holder on a Vortex-Genie at maximum speed; after steep 3, pipette $550 \mu \mathrm{l}$ of clean supernatant in to a new $2 \mathrm{ml}$ Eppendorf, centrifuge again at 20,000 g for $2 \mathrm{~min}$ and continue with steep 4; in steep 6, centrifuge for $3 \mathrm{~min}$; in steep 7 , add $50 \mu \mathrm{l}$ of $\mathrm{ddH}_{2} \mathrm{O}$ and incubate for $3 \mathrm{~min}$.

\section{3 | High-throughput sequencing}

Both dropping and sweeping samples were sequenced in the Bart's and the London Genome Centre, London, UK. DNA extracts were checked for quality and concentration on a TapeStation D1000. Two sets of primers were used together in order to reduce primer taxonomic bias (Alberdi et al., 2018), especially given the high diversity of prey types expected in the diet, ZBJ (Zeale et al., 2011) Forward: ZBJ-ArtF1c 5'-AGATATTGGAACWTTATATTTTATTTTTGG-3' and Reverse: ZBJ-ArtR2C 5'-WACTAATCAATTWCCAAATCCTCC-3', and ANML (Jusino et al., 2019) Forward: LCO1490 5'-GGTCAACAAATCATAAAGATATTGG-3' and Reverse: CO1CFMRa 5'-GGWACTAATCAATTTCCAAATCC-3'. For the ZBJ amplicon each $15 \mu \mathrm{l}$ PCR reaction used $7.5 \mu$ of Multiplex PCR mastermix (QIAGEN, Germany), $0.25 \mu \mathrm{L}$ of each primer $(10 \mu \mathrm{M}), 5 \mu \mathrm{L} \mathrm{H} 2 \mathrm{O}$ and $2 \mu$ template DNA. Negative and positive controls were included in $\mathrm{PCR}$ reactions and later sequencing. The thermal cycling protocol was as follows: $95^{\circ} \mathrm{C}$ for $15 \mathrm{~min}, 34 \mathrm{cycles}$ of $94^{\circ} \mathrm{C}$ for $40 \mathrm{~s}, 40^{\circ} \mathrm{C}$ for $1 \mathrm{~min}, 72^{\circ} \mathrm{C}$ for $30 \mathrm{~s}$, followed by a final extension of $72^{\circ} \mathrm{C}$ for $5 \mathrm{~min}$. ANML regions were amplified in $15 \mu$ reactions following published protocols (Jusino et al., 2019). All products were visualized on a $1.5 \%$ agarose gel. Products were tagged using Fluidigm barcodes and checked on a TapeStation D1000 before pooling and sequencing on an Illumina MiSeq using paired end $(2 \times 250$ bp) chemistry (Illumina, San Francisco, USA). We used two technical PCR replicates to reduce biases associated with PCR stochasticity. This led to each sample being sequenced four times (combination of two primer and two PCR replicates).

\subsection{Bioinformatics}

Sequencing runs were merged using USEARCH (Edgar, 2010) and primers and adaptors removed using cutadapt (Martin, 2011). Sequences were processed on the mBRAVE platform (http://www. boldsystems.org/bin) (Ratnasingham \& Hebert, 2007) setting the following parameters: Min QV $=0$ qv, Min Length $=100$ bp, Max Bases with Low QV $(<20)=75 \%$, Max Bases with Ultra Low QV $(<10)=75 \%$, ID Distance Threshold $=1.5 \%$, Exclude from OTU Threshold $=3 \%$, Minimum OTU Size $=1$, OTU Threshold $=2 \%$. Sequences were compared with the BOLD reference libraries SYS-CRLINSECTA and SYS-CRLNONINSECTARTH to established
Barcode Index Numbers (BINs). BINs are a type of Operational Taxonomic Unit (OTU) integrated in the BOLD system with advantages over traditional OTUs, such as being unique and stable (Ratnasingham \& Hebert, 2013). We used BIN identity as a proxy of taxonomic prey item unit.

After obtaining BIN (prey item) composition per sample and run, we removed singletons, that is, BINs that only had a single read per run and sample, because they are likely to be PCR or sequencing errors (Alberdi et al., 2018). We set the threshold for the minimum number of reads needed to be present in each sample to retain a BIN identification to two (singletons removed) because this threshold resulted in highest similarity in arthropod composition in sweeping samples between molecular and morphological identification methods (range of thresholds tested: 1-5 minimum reads/sample). We controlled for potential contamination during the extraction and sequencing process by removing the BINs present in extraction/sequencing blanks from samples in the same extraction/sequencing run that had a similar number of these reads to the blanks (less than 10 times more reads in samples than in blank). To assess impact of BIN removal, all analyses were run without removing the BINs potentially resulting from contamination. We found that BIN removal did not affect the study results.

We used two alternative approaches to combine data from PCR replicates. In the first, the additive criteria, BIN composition from both PCR replicates of each sample were added together. In the second, the conservative approach, only BINs that appeared in both PCR replicates were considered (Alberdi et al., 2018). Under this second criteria only samples in which the four runs contained a minimum number of reads $(>100)$ were considered given that a failed sequencing run in a sample would lead to a null composition for both of the PCR replicates of a primer (52 samples, Table S3). Finally, we combined taxa recovered from both primers to obtain the prey composition per dropping sample for downstream ecological analysis. Duplicated BINs in the same sample coming from different PCR replicates or primers were removed. A flow chart describing the methods is shown in Fig. S1. Ecological results from both approaches were very similar, thus we present results based on the additive approach (See Fig. S9 for diet based on the conservative approach).

\section{5 | Characterizing the diets of the two bat species}

The contribution of different elements to the diet for a set of samples was quantified using weighted Percent of Occurrence (wPOO), which measures the relative occurrence of diet elements (prey items/OTUs/BINs) in a set of samples considering first their relative proportion per sample (Deagle et al., 2019). For example, a prey item found in a sample with 9 other prey items will be interpreted to contribute in the diet $1 / 10$ of what it would if it was the only prey item present. Contributions to diet based on the two other commonly used metrics: Percent of Occurrence (POO) and Relative Read Abundance (RRA) (Deagle et al., 2019), are shown in 
(Fig. S2). We tested for differences in the number of BINs per sample between bat species for each of the orders that constitute at least $10 \%$ of the diet of either one of the bat species (Araneae, Diptera, Lepidoptera, and Hemiptera), using negative binomial generalized linear models (GLMs; in R) to fit data structure based on the distribution of model residuals. We measured order level and prey species level (BIN-level) diet composition overlap between bat species using Pianka's measure of niche overlap $\left(\mathrm{O}_{\mathrm{jk}}\right)(\mathrm{R}$ package: EcoSimR (Gotelli et al., 2015). We tested with an ANOSIM test (R package vegan (Oksanen et al., 2019)) whether Jaccard distance in BIN composition was greater between than within bat species. The ANOSIM statistic $R$ is based on the difference of mean ranks between and within groups, with a range between -1 and +1 . A value of zero indicates that the group does not explain compositional differences. We visualized ordination of samples depending on their BIN composition with Non-Metric Multidimensional Scaling (NMDS, R package vegan: Oksanen et al., 2019). We calculated Levins' (1968) standardized measure of niche breadth $\left(B_{A}\right)$ at the prey species (BIN) level for each bat species.

\subsection{Functional diet assessment}

Prey items were classified based on the literature (outlined in Table S2) and an expert entomological taxonomist into three functional categories: non-volant, not actively volant, nocturnally volant. Categorization depended on their mobility and type of activity, reflecting their likelihood of being captured by gleaning or aeria hawking (Data file S1, Table S2). The categorization was done at family or finer taxonomic level by checking the literature for data on daily activity patterns of each family and presence in nocturnal light traps (Table S2 for criteria used). The non-volant category included wingless arthropod groups (Araneae, Isopoda and wingless insects such as some members of Blattodea, Orthoptera). The not actively volant category included those able to fly but unlikely to have been captured by the bat through aerial hawking because they are not active fliers, either at night (diurnal Diptera), or not active fliers in general (e.g., Hemiptera, some Blattodea, Orthoptera and Coleoptera). The nocturnally volant category comprised arthropods with aerial and nocturnal activity and therefore likely to be captured by aerial hawking (e.g., non-Ropalocera Lepidoptera, nocturnal Diptera, Neuroptera, Ephemeroptera, Trichoptera). This classification represents the likelihood of being captured by gleaning or aerial hawking rather than direct inference of the capture mode because nocturnally active aerial prey can also be captured by gleaning when resting on vegetation and not active nocturnal fliers could also be captured in the air (e.g., ballooning in spiders). Once all prey items were classified into functional groups, we obtained the functional diet of both bat species using weighted percent of occurrence (wPOO), and compared the percentage of not nocturnally volant (including both non-volant and not actively volant categories) per sample between bat species using a linear model.

\section{7 | Trophic niche overlap in allopatry versus sympatry across spatial scales}

Locations from Andalusia (Mediterranean climate) and northern Cantabria (Atlantic climate) were classified as regionally allopatric. Locations from La Rioja and southern Cantabria (climatically Mediterranean to sub-Atlantic) as regionally sympatric (based on data from Razgour et al., (2019) and EBD records). At the fine-scale within the sympatric regions, we classified locations as syntopic (both species co-occurring) or allotopic (species not co-occurring) depending on whether they were within $3 \mathrm{~km}$ of records of the other species. Distances were set based on a conservative estimation of the home-range distance of the better studied cryptic congener M. nattereri (Boye \& Dietz, 2005). The swarming location in Catalonia was removed from the fine-scale analysis because bats gather in swarming sites from distances of up to $60 \mathrm{~km}$ from their colonies for the purpose of breeding rather than foraging (Rivers et al., 2005), and therefore, it is unclear whether those individuals forage in sympatry (Table S1 for sampling locations and their broad and fine-scale sympatry category).

To identify differential use of certain prey orders and functional groups, we tested separately for allopatry and sympatry whether (a) the number of BINs per sample for each of the main arthropod orders, and (b) the percentage of not nocturnally volant functional groups differed between bat species. We used negative binomial zero inflated GLMs and a linear model respectively. We run separate models for broad and fine spatial scales given that both syntopy and allotopy treatments are within regional sympatry. Cases where resource (prey order or functional group) use was different between bat species when sympatric but not when allopatric were regarded as evidence of resource partitioning. We measured prey species (BIN) level niche overlap $\left(\mathrm{O}_{\mathrm{jk}}\right)$ between bat species in sympatry and in allopatry, and tested, using null models ( $R$ package eco$\operatorname{simR}$ ) whether overlap was lower or higher than random in sympatry versus allopatry. We tested whether $\mathrm{O}_{\mathrm{jk}}$ differed between sympatric and allopatric regions and syntopic and allotopic locations by pooling the diet composition of each bat species per site and measuring $\mathrm{O}_{\mathrm{jk}}$ between pairs of sites. At the regional scale we used a Gaussian Hurdle model due to the high density of zeroes in overlap values. At the local scale we used a linear model with log transformed values of $\mathrm{O}_{\mathrm{jk}}$ to meet assumptions of normal distribution. All statistical analysis was carried out in R ( $R$ core team, 2020).

\subsection{Prey consumption relative to availability}

To assess the representativeness of arthropod availability sampling, we checked for each dropping sample the proportion of BINs that appear in the sweeping samples from the same site. We retained only the sites where prey availability sampling was considered most representative because at least $20 \%$ of the diet prey items in the site were found in sweeping samples. For each of these sites, we quantified the relative availability of each 
arthropod order and functional group using weighted percent of occurrence (wPOO) after pooling together sweeping samples from the different habitats. Similarly, we obtained bat diet composition (WPOO) per site for each arthropod order and functional group by pooling diet composition of all individual bats. Then, we subtracted from the bat diet wPOO the prey availability wPOO to obtain prey arthropod and functional group selection per site. A higher proportion of a given arthropod order in the diet than in sweeping samples indicates the bats may be preferentially consuming this resource, based on prey availability at the sampled strata. We calculated whether confidence intervals of selection values across sites are above zero.

\section{9 | Testing primer performance and representation of the DNA metabarcoding approach}

For each arthropod order we described the number and proportion of BINs identified by each primer. Morphological identification of the arthropod communities allowed us to compare the performance of the primers and metabarcoding approaches. We compared the presence of orders in each sweeping sample based on molecular and morphological identification to determine whether metabarcoding offers a good estimation of arthropod community composition.

\section{3 | RESULTS}

We analyzed a total of 138 dropping samples for Myotis escalerai and 90 for Myotis crypticus from 49 locations, 26 of which were in the broad-scale allopatric regions and 23 in sympatric regions. Within the sympatric regions (La Rioja and southern Cantabria), 91 samples were classified as allotopic and 28 as syntopic (Figure 2, Table 1; Table S1). Sample sizes were limited by the small number of known syntopic locations for these species. We recovered a total of $2,859,300$ reads (Table S3 for details) from the 228 dropping samples for the four combinations of PCR replicates and primers $(1,403,636$ from ANML1 and 1,455,664 from ZBJ). These reads were associated into 1,461 different BINs, 941 for M. escalerai and 851 for M. crypticus. Based on BINs present in extraction blanks, we removed a total of 8 BINs from 10 dropping samples (Table S4). Based on the BINs present in sequencing blanks, we removed for the ANML primers 6 BINs from 66 dropping samples, and for the ZBJ primers, 24 BINs from 95 samples (Table S5).

\subsection{Characterizing the diet of M. escalerai and M. crypticus}

A total 19 arthropod orders were obtained based on the 1,461 BINs (Supplementary Data file S1 for list of prey items obtained for each bat species). The diets of M. escalerai and M. crypticus were characterized by high arthropod diversity, and were composed mostly of the orders Lepidoptera (M. escalerai $=26.6 \%$ wPOO; M. crypticus = 23.7\%), Diptera (24.8\%; 33.2\%), Araneae (20.7\%; 17.2\%), but also included Hemiptera (11.8\%; 6.2\%), Coleoptera (4.8\%; 5.1\%), and Orthoptera (4.3\%; 4.8\%), among others (<5\%) (Figure 3a-b; Fig. S2 for diet composition based on POO and RRA measures). The most common prey species consumed by $M$. escalerai were Philodromus dispar, Xysticus ferrugineus/audax, Chorthippus vagans, Metasia sp., and Ectobius pallidus, while the most common prey species consumed by M. crypticus were Philodromus dispar, Ectobius pallidus, Araneus diadematus, Delia platura, and Chorthippus vagans. Hence, the two bats shared three of the top five common prey species. Diet composition at the prey order level was very similar between bat species $\left(\mathrm{O}_{\mathrm{JK}}=0.98\right.$, above 1,000 null models). However, there were differences in the number of BINs per sample of Diptera, which was lower in M. escalerai (5.27 versus 6.75) (Negative binomial GLM: $z_{1,226}=-2.03, p=.042$ ), and Hemiptera, which was higher in M. escalerai (2.09 versus 1.68) (Negative binomial GLM: $z_{1,226}=2.85, p=.004$ Fig. S3).

At the prey species (BIN) level, Levins' niche breadth was similar for both species, $B_{A}=0.17$ for $M$. escalerai and $B_{A}=0.19$ for M. crypticus. Niche overlap between species was higher than expected by chance $\left(\mathrm{O}_{\mathrm{JK}}=0.71\right.$, above $95 \%$ of 1,000 null models). The samples from the two bat species showed some differences in prey item composition in NMDS ordination space (Figure 4a, Stress: 0.25, $k=3$, non-metric fit $R^{2}=0.934$, Linear fit, $R^{2}=0.532$ ). An analysis of similarity confirms that distance in prey item composition among samples is greater between species than within species (ANOSIM R statistic: $0.10, p=.001$ ).

TAB LE 1 Number of bat dropping samples, sweeping samples, and locations for each bat species by allopatry/sympatry classification at broad (regional) and fine (local) spatial scales

\begin{tabular}{|c|c|c|c|c|c|c|}
\hline & & Total & Broad-scale allopatric & $\begin{array}{l}\text { Broad-scale } \\
\text { sympatric }\end{array}$ & Allotopic & Syntopic \\
\hline Dropping samples & M. escalerai & 138 & 82 & 56 & 46 & 6 \\
\hline \multirow[t]{2}{*}{ Sweeping samples } & M. escalerai & 13 & 5 & 8 & 3 & 5 \\
\hline & M. crypticus & 15 & 2 & 13 & 6 & 7 \\
\hline
\end{tabular}


(a) Diet Myotis escalerai (wPOO)

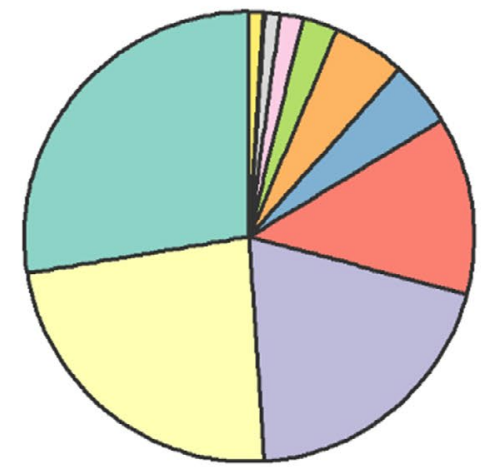

(c)

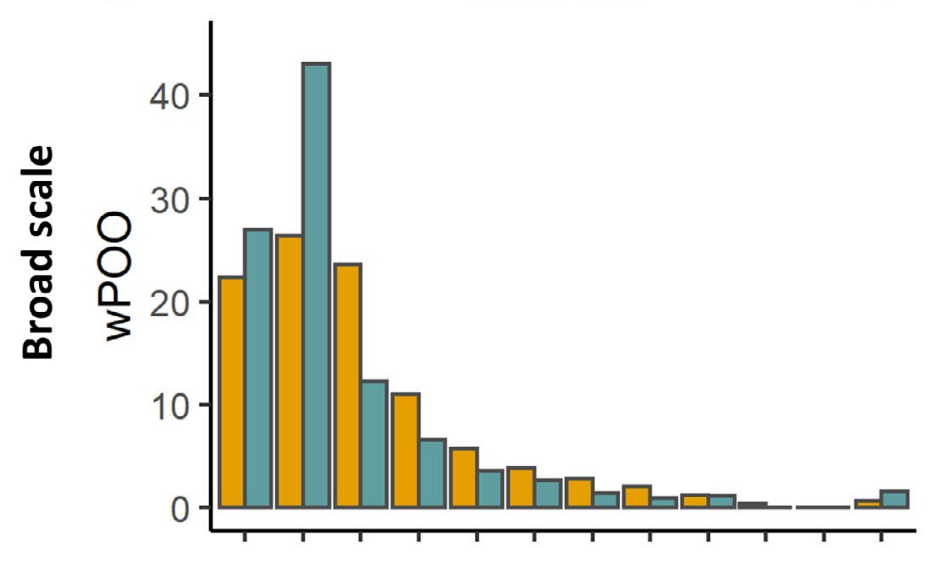

(e)

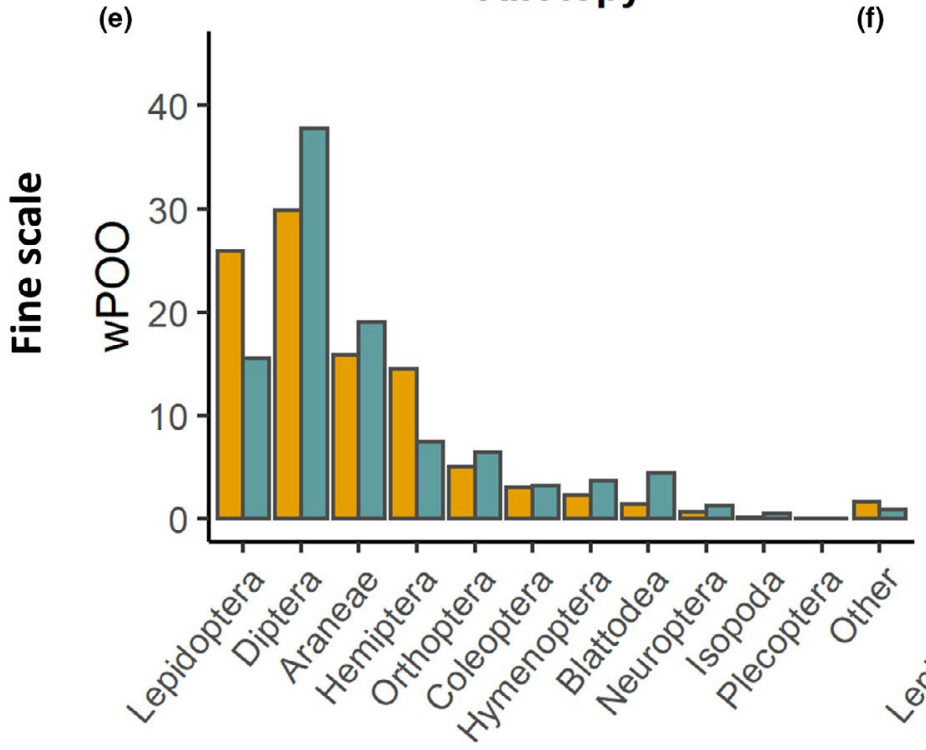

(d) (b) Diet Myotis crypticus (wPOO)

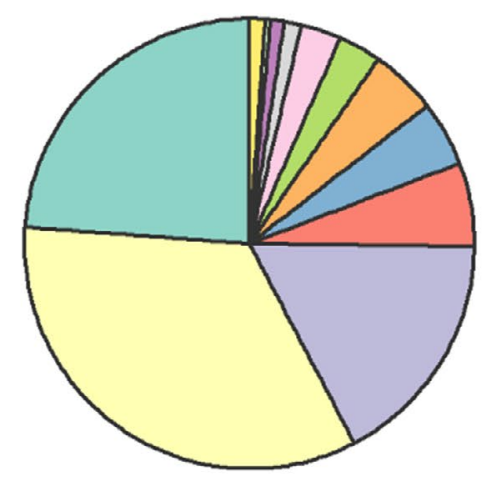

Arthropod orders
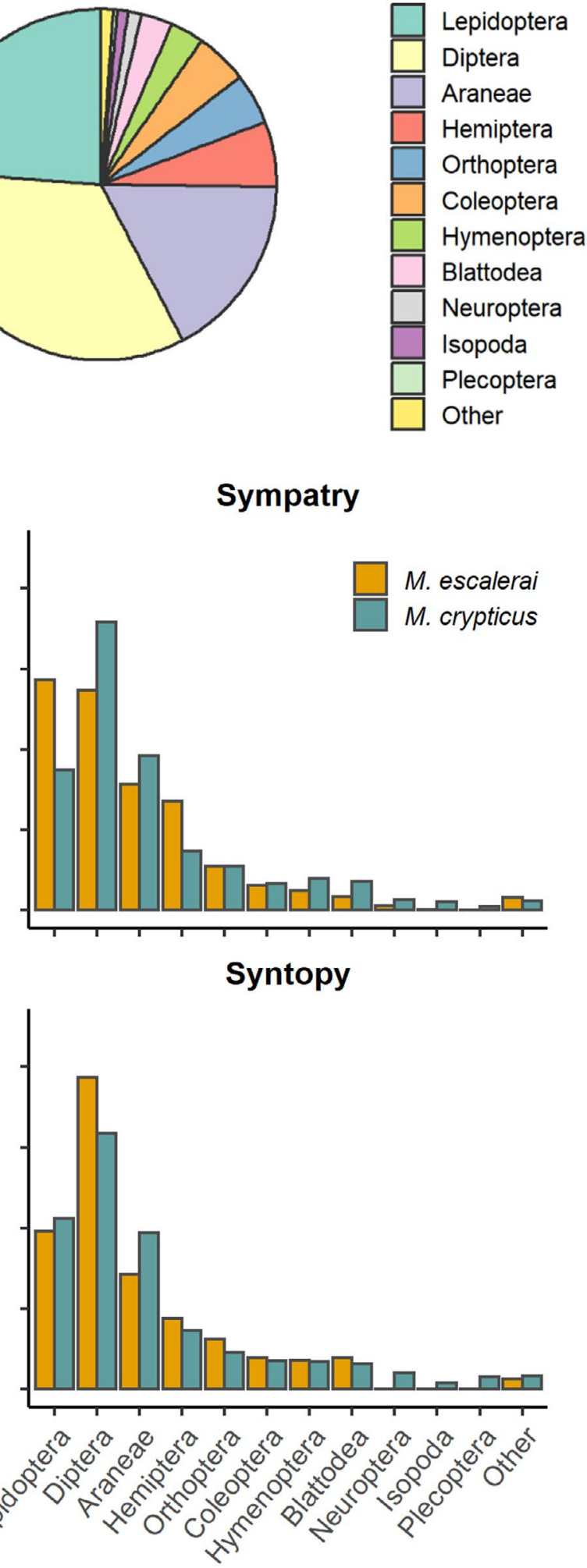

FIGURE 3 Overall diet composition ofM. escaleraiandM. crypticususingweighted Percent of Occurrence (wPOO) (a, b). Dietary composition by scale of allopatry/sympatry (broad-scale: c, $d$, fine-scale: e, f) 

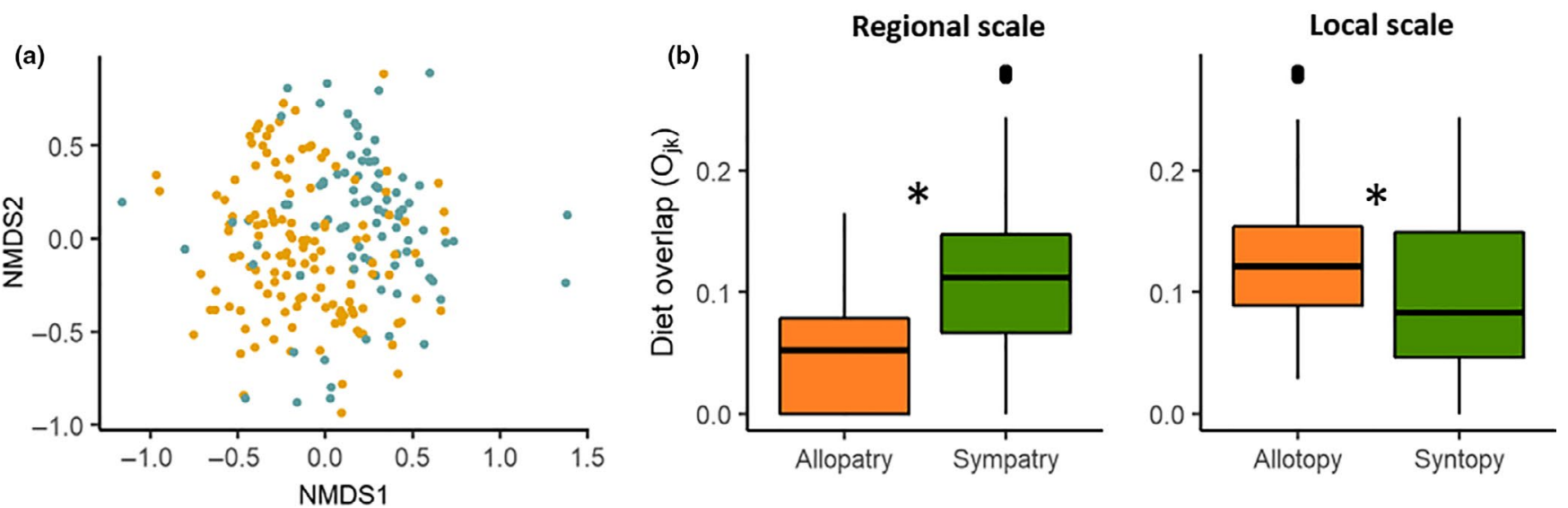

FIGURE 4 (a) Non-Metric Multidimensional Scaling ordination of individual bat samples based on their BIN composition, withM. escaleraisamples in yellow andM. crypticusin blue. (b) Pianka's measure of niche overlap $\left(\mathrm{O}_{\mathrm{JK}}\right)$ between the two bat species in allopatric versus sympatric locations at the regional (left) and local (right) scales. Replicates are values of overlap between pairs of locations of different bat species. Star denotes significant differences between groups

\subsection{Trophic partitioning in sympatric versus allopatric locations}

At the arthropod order level, there is no clear pattern of shift from high similarity in order composition between species to differential use in sympatry at any of both spatial scales $\left(\mathrm{O}_{\mathrm{JK}}\right.$ regional allopatry $=0.88$, $\mathrm{O}_{\mathrm{JK}}$ regional sympatry $=0.96,>1,000$ null models, Figure $2 \mathrm{c}-\mathrm{d}$; $\mathrm{O}_{\mathrm{JK}}$ allotopy $=0.95, \mathrm{O}_{\mathrm{JK}}$ syntopy $=0.98,>1,000$ null models, Figure $3 \mathrm{e}-\mathrm{f}$ ). When examining the number of BINs of the main arthropod orders per sample, there were differences between bat species between the allopatric regions for Araneae and Hemiptera, which were both higher in M. escalerai (M. escalerai $=4.00,1.65$, M. crypticus $=2.16,0.55$ respectively), and for Lepidoptera, which was higher in M. crypticus $(4.6,11.94)$ (Negative binomial GLM: $d f=1,98, p<.05$ ). In the sympatric region, the higher number of Hemiptera in M. escalerai holds (M. escalerai $=2.50$, M. crypticus 1.50), and in Lepidoptera there is a shift whereby is M. escalerai the one that consumes a higher number ( $M$. escalerai $=6.78$, M. cryptius $=4.09$, Negative binomial GLM: $p<.05)$. At the fine-scale, within the sympatric region, the only difference found between the bat species was the higher number of BINs per sample of Hemiptera (2.67, 1.49) (Negative binomial GLM: $z_{1,89}=-2.68, p=.007$ ) and Lepidoptera $(6.70,3.64)$ in M. escalerai in allotopic locations (Negative binomial GLM: $\left.z_{1,89}=-2.92, p=.004\right)$. There were no differences in arthropod orders consumed between the bat species in syntopic locations (Negative binomial GLM: $p>$.05; Fig. S4).

At the prey species (BIN) level, at the broad-scale, trophic niche similarity between species was lower in allopatric than in sympatric regions $\left(\mathrm{O}_{\mathrm{JK}}\right.$ allopatric $=0.35, \mathrm{O}_{\mathrm{JK}}$ sympatric $\left.=0.62\right)$. Conversely, at the fine-scale, within the sympatric region, trophic niche overlap between species was higher in allotopic locations $\left(\mathrm{O}_{\mathrm{JK}}=0.56\right)$ than in syntopic locations $\left(\mathrm{O}_{\mathrm{JK}}=0.37\right)$. However, in all the four cases, observed niche overlap between species was higher than 1,000 null models. When measuring trophic niche overlap between species using pairs of locations, we observed the same pattern. At the broad scale, we found higher diet overlap in sympatry than allopatry $\left(\mathrm{O}_{\mathrm{JK}}\right.$ sympatric $=0.107 \pm 0.056, \mathrm{O}_{\mathrm{JK}}$ allopatric $=0.050 \pm 0.04 ;$ Gausian hurdle model: binomial GLM: $z_{1,316}=4.76, p<.05$; Gaussian GLM: $\left.t_{1,265}=8.26, p<.05\right)$. In contrast, at the fine-scale, niche overlap was lower among pairs of syntopic than allotopic locations $\left(\mathrm{O}_{\mathrm{JK}}\right.$ syntopic $=0.099 \pm 0.065, \mathrm{O}_{\mathrm{JK}}$ allotopic $=0.126 \pm 0.057$; Linear model: $F_{1,73}=6.34, p=.014$; Figure $4 b$ ).

\section{3 | Functional diet analysis}

Both species had a similar high percentage of non-volant (M. escalerai $=21.4 \%$, M. crypticus $=19.5 \%)$ and not actively volant $(44.6 \%$, $45.8 \%$ ) prey items in the diet. Only $34.0 \%$ and $34.7 \%$ of weighted percent of occurrence (wPOO) was composed of arthropods classified as nocturnally volant (Figure $5 \mathrm{a}$ ). There were no differences in the overall percentage of not nocturnally volant prey taxa (BINs) per sample between bat species $(66 \% \pm 20 \%, 66 \% \pm 21 \%$, Linear model: $F_{1,217}<0.001, p=.990$; Figure $\left.5 b\right)$. When analyzing functional diet differences separately in allopatric versus sympatric regions, we found differences between species in allopatric regions, whereby M. crypticus consumed lower percentage of prey that were not nocturnally volant (allopatric regions: M. escalerai $=66 \% \pm 19 \%$, M. crypticus $=48 \% \pm 25 \%$; $F_{1,98}=11.72, p<.05$; Figure $5 c$; sympatric regions: $65 \% \pm 22 \%, 71 \%$ $\pm 17 \% ; F_{1,117}=2.3, p=.13$; Figure $5 d$ ). At the fine-scale, there were no differences among bats in allotopic locations (M. escalerai $=67 \% \pm 22 \%$, M. crypticus $=72 \% \pm 16 \%, F_{1,89}=1.325, p=.250$; Figure $5 \mathrm{e}$ ) while in syntopic locations the percent of prey that were not nocturnally volant was borderline lower in the diet of M. escalerai $(52 \% \pm 17 \%)$ than M. crypticus $\left(68 \% \pm 18 \% ; F_{1,26}=4.03, p=.055\right.$; Figure $\left.5 f\right)$.

\subsection{Prey consumption relative to availability}

The proportion of BINs consumed by the bats that were also present in the sweeping samples of their respective capture sites was 
(a)

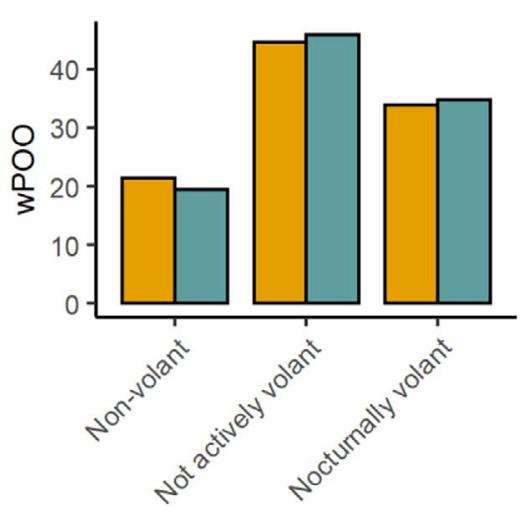

(c)

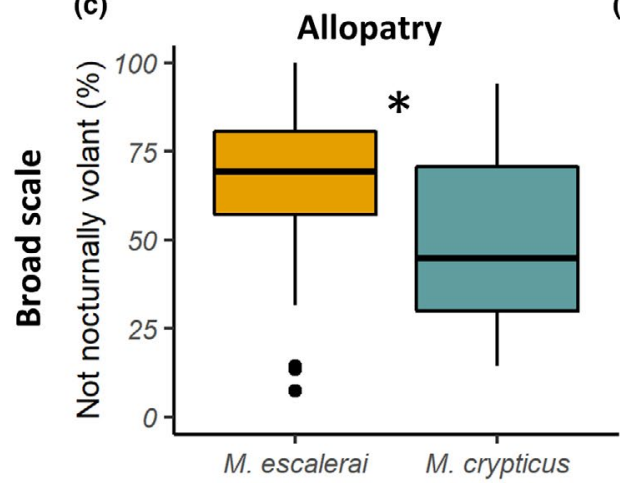

(e)

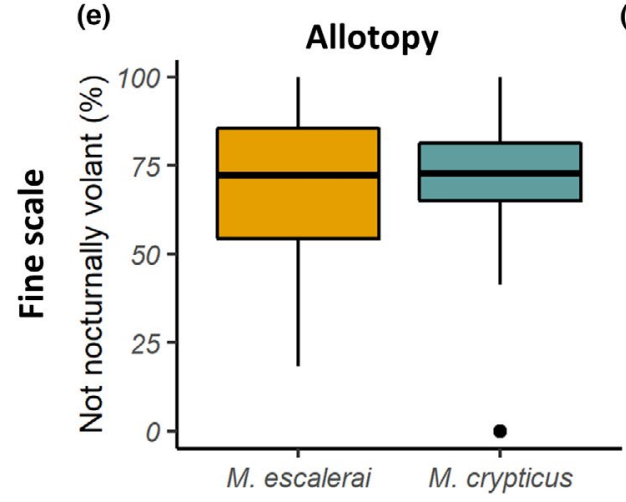

(b)

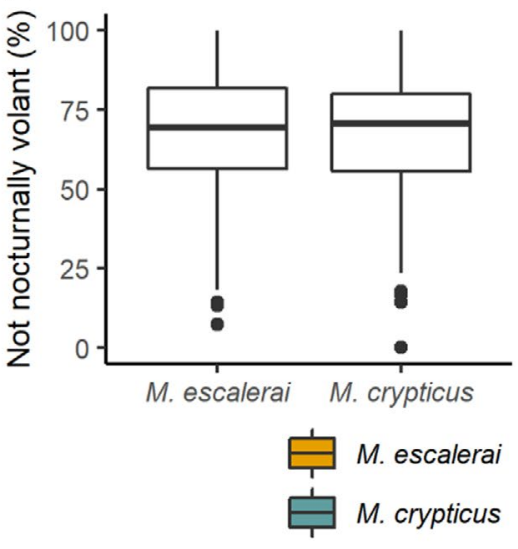

(d)

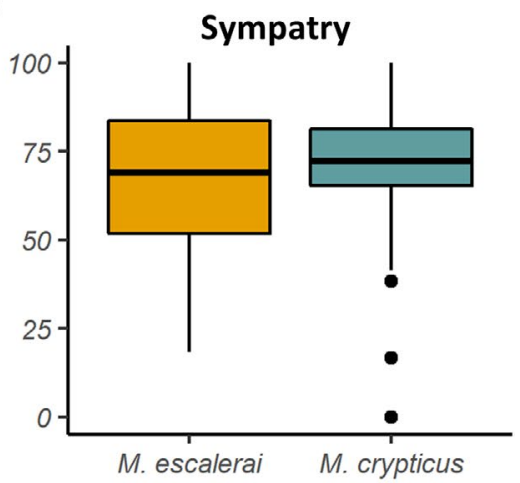

(f)

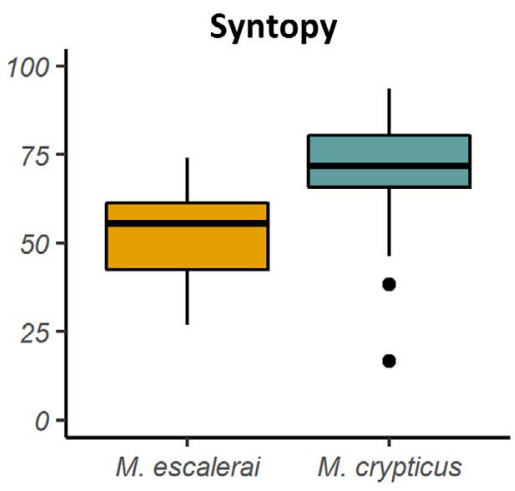

FIGURE 5 Functional diets ofM. escaleraiandM. crypticusdepending on the nocturnal flight behavior of the prey species, classified into non-volant (wingless arthropod groups), not actively volant (able to fly but unlikely to have been captured through aerial hawking), and nocturnally volant (likely to be captured by aerial hawking). Overall proportions of functional categories in the bat diets (a); proportion of not nocturnally volant prey items per dropping sample inM. escaleraiandM. crypticusoverall (b), in broad-scale allopatric versus sympatric regions (c) and in fine-scale allotopic versus syntopic locations (d). Star denotes significant differences between groups (Linear Model) highly variable but generally low. In the dropping samples of five sites $<10 \%$ of the BINs on average also appeared in the sweeping samples, in nine sites $10 \%-20 \%$, in seven sites $20 \%-30 \%$ and only for two sites $>30 \%$ of the BINs appeared in the sweeping samples. We retained only the nine sites where at least $20 \%$ of the BINs in the bat diet were present in sweeping samples. We detect underselection by both species for Diptera, with the distribution of selection values between the 1 st and 3 rd quartiles falling below zero (1st-3rd quartiles M. escalerai: -16.24 to -1.01 , M. crypticus: -24.40 to 9.55), and over-selection by both species for Lepidoptera, with the distribution of selection values between the 1st and 3rd quartiles falling above zero (1st-3rd quartile M. escalerai: 2.04-2.49, M. crypticus: $4.30-12.41$ ). In the other two main orders (Areanea and Hemiptera), 1st and 3rd quartiles of selection values overlapped zero (Fig. S5).

\subsection{Metabarcoding and primer performance}

There were compositional differences in the prey orders that each primer recovered. A large proportion of the BINs identified in dropping samples were only recovered by one of the primers. Neuroptera, Orthoptera, and Coleoptera were more frequently recovered by ZBJ, while Plecoptera, Thysanoptera, Dermaptera, and Mantodea were more frequently recovered by ANML (Fig. S6). Figure S7a-b shows composition for a subset of dropping samples comparing each primer.

In sweeping samples, 7,065 insects were identified morphologically to order level, with an average of 174.3 individuals per sample (range 7-624). Using molecular tools, we recovered 899,853 reads (Table S2), and identified 813 different BIN items. Some of the rarer orders were under-represented in the molecular analysis. 
Specifically, Opiliones, Dermaptera, and Archaeognatha appeared in more than 10 sweeping samples each identified morphologically, but were rarely recovered in the molecular approach, despite being present in the reference databases (Fig. S8).

\section{4 | DISCUSSION}

Morphologically almost identical species are likely to compete for resources (Wainwright \& Reilly, 1994) and therefore offer a good study system to understand processes that drive species coexistence. If trophic resource partitioning enables species coexistence, trophic similarity is expected to be lower when in sympatry. We find that despite overall very high trophic similarity among the recently separated cryptic bats M. escalerai and M. crypticus, trophic niche overlap is lower in syntopic relative to allotopic locations. The functional analysis suggests that the subtle trophic shift seen may be driven by differential foraging mode. Our results support niche theory predictions of the role of biotic interactions in driving species assemblages (Schoener, 1974). Trophic resource partitioning was only detected at the fine spatial scale in locations where the two species are syntopic and therefore more likely to compete for resources.

\subsection{Trophic ecology of Myotis escalerai and Myotis crypticus}

Our results reveal that the two bat species have a broad generalist diet and use gleaning to a high extent. We found a very high similarity in their trophic ecology in terms of both order and prey species composition. Both bat species' diets are mostly composed of Lepidoptera, Diptera and Araneae, but also include several other prey orders. However, M. escalerai consumes a higher percentage of Hemiptera, while M. crypticus Diptera. Functionally, the two bat species consume an equally high proportion of prey items that are not nocturnally volant, which suggests that both bats predominantly glean prey from vegetation.

The trophic ecology of these two recently described bat species is very similar to their cryptic sister-species M. nattereri, which also feeds mostly on Lepidoptera, Diptera and Araneae (Hope et al., 2014; Swift, 1997; Swift \& Racey, 2002; Vaughan, 1997) and is known to catch a high proportion of its prey through gleaning (Arlettaz, 1996; Hope et al., 2014; Shiel et al., 1991; Swift, 1997; Swift \& Racey, 2002). Similarly to our study, Shiel et al. (1991) estimated that $68 \%$ of $M$. nattereri's diet is made up of arthropod families that are not active at night. Although the characteristic row of hairs in the uropatagium border of the M. nattereri species complex is thought to be functionally linked with gleaning (Czech et al., 2009) and the presence of more developed hairs in M. escalerai is one of the few characteristics which separates these taxa (Juste et al., 2019), we found no difference in the extent of gleaning between the two bat species. This study confirms that the previously identified high proportion of spiders in the diet of $M$. nattereri is common to the rest of the cryptic complex. Spiders are a rare component of the diet of most European bat species, with the exception of Myotis emarginatus (Vallejo et al., 2019).

\section{2 | Trophic partitioning across spatial scales}

At the prey order level, niche similarity between the two bat species was overall high. However, at the prey species (BIN) level, we detect a signature of trophic shift, whereby diet overlap is lower in syntopic compared to allotopic locations at the fine-scale. This supports the contribution of trophic partitioning to species coexistence even when overall trophic niche overlap is high, though given the high trophic similarity among the two bat species, other mechanisms of resource partitioning, such as spatial and microhabitat partitioning, likely also play an important role. A similar trend is observed at the functional level, whereby the proportion of prey items that are not nocturnally volant is borderline different between bat species only when syntopic. This suggests that the differentiation in diet composition seen at the prey species level when in syntopy may be driven by a shift in foraging strategy (e.g., Krüger et al., 2014), through M. escalerai decreasing its extent of gleaning. However, our inference is limited by small sample sizes, which reduced the power of the analysis. At the arthropod order level, we find differences in the use of some arthropod orders among allopatric regions, likely due to differences in arthropod availability between the Mediterranean region, where only M. escalerai is found and the Atlantic region, where M. crypticus is present.

Several studies have identified trophic niche shifts from allopatry to sympatry, for instance between morphologically similar fish (Gkenas et al., 2019; Schmitt \& Coyer, 1983) and reptile species (Huey et al., 1974; Klawinski et al., 1994). However, in bats, previous coexistence studies looking at trophic ecology only focused on sympatric or syntopic populations, and rarely found evidence of trophic resource partitioning. A few exceptions are the gleaning bats M. nattereri, Plecotus auritus, and Myotis bechsteini in a shared roost in central Europe (Andreas et al., 2012a), and evidence of low dietary overlap between co-occurring P. auritus and Plecotus macrobullaris (Ashrafi et al., 2011).

The observed trophic shift, albeit subtle, suggests that the two bat species are likely competing for food resources. It has been previously hypothesized that arthropods are abundant and do not constitute a limiting resource for bats (Arlettaz, 1999; Krüger et al., 2014). However, exclusion experiments in both tropical (Kalka et al., 2008) and temperate forests (Böhm et al., 2011) show that bats can control the abundance of arthropods, and therefore, arthropods could be a limiting resource to competitors (SalinasRamos et al., 2020).

Our study does not refute the possibility that other coexistence mechanisms, such as habitat or temporal partitioning (Schoener, 1974), occur among these two species, or the role of 
environmental variability in facilitating coexistence (Chesson \& Warner, 1981). Spatial partitioning is frequently cited as a key mechanism of coexistence in other bat studies (e.g., Arlettaz, 1999; Emrich et al., 2014; Kunz, 1973; Russo et al., 2014). Although in many cases, contrary to our study, spatial partitioning may be driven by slight differences in bat morphology (e.g., Salsamendi et al., 2008, 2012), which would affect their performance in different habitats (Norberg, 1994). However, in our study the two species were caught in the same sampling sites, some of which were forests, where they are known to forage (Juste et al., 2019), suggesting they may share the same foraging sites.

Our finding that trophic partitioning only occurs at the fine spatial scale is consistent with other studies of bats (Peixoto et al., 2018), ants (Albrecht \& Gotelli, 2001), parasitoid insects (Harvey et al., 2014) and bobcats (Lewis et al., 2015), showing that interspecific interactions are more important for shaping community structure at fine rather than broad spatial scales. However, this pattern is not universal (e.g., Harmáčková et al., 2019). In our study system, these fine-scale mechanisms could contribute to enabling broad-scale range overlap across the north of the Iberian Peninsula because theoretical studies have shown that fine-scale coexistence mechanisms can prevent competitive effects from scaling-up and affecting the broad-scale distributions of species (Godsoe et al., 2015).

\subsection{Prey consumption relative to availability}

The diet of a species is a function of both consumer selection and trophic resource availability within the foraging habitat (Lawlor, 1980). Therefore, considering resource availability allows for a better inference of species trophic preferences. Previous studies comparing bat prey consumption with prey availability pointed to selection of certain prey orders, such as Coleoptera by Eptesicus fuscus (Agosta et al., 2003), chironomid flies by Myotis daubentonii (Vesterinen et al., 2016) and certain prey traits like moth size by Barbastella barbastella (Andreas et al., 2012b). Similarly, M. nattereri was found to over-select arachnids, Opiliones, Coleoptera, and several Diptera families, and under-select Hemiptera (Swift \& Racey, 2002). In this study we detect over-selection of Lepidoptera and under-selection of Diptera by both bat species. However, diet selection results should be interpreted with caution. Any arthropod sampling technique is biased toward certain types of arthropods (Cooper \& Whitmore, 1990). Our molecular diet analysis results confirm that the two bat species glean prey from the vegetation, and therefore, sweep nets are the appropriate arthropod sampling method to study prey availability. However, the low proportion of BINs in bat diet that appear in sweeping samples does not support the representativeness of our prey availability sampling, likely due to insufficient sampling effort or inadequate coverage of all the areas where the bats forage. This is because bats can use large areas and arthropod communities change depending on habitat type (Lamarre et al., 2016) and vertical stratification (Ulyshen, 2011).

\subsection{Methodological considerations and study limitations}

Primer bias toward certain taxonomic groups is a major issue in metabarcoding studies (Elbrecht et al., 2019). In this study, prey items were frequently recovered by only one of the primers, and differences existed in the recovery of the different arthropod orders. This supports previous studies that suggest that more than one set of primers should be used when the expected diet covers a broader taxonomical spectrum (Alberdi et al., 2018; Aldasoro et al., 2019; Esnaola et al., 2018). The inclusion in this study of a set of samples with known composition based on morphological analysis (albeit only at the order level) gives us some idea of potential biases in the molecular identification. Opiliones, in particular, were morphologically identified in several sweep net samples and are known to be present in the diet of M. nattereri (Galan et al., 2018; Swift, 1997; Swift \& Racey, 2002), but were absent from the molecularly characterized diets of the two bats. Thus, their absence in this study is likely the result of primer amplification bias.

Because prey development stage cannot be identified using the metabarcoding approach, some of the prey species (BINs) classified as nocturnally volant may correspond to non-flying larval stages. This could be important in Lepidoptera, and could increase the inferred importance of the gleaned behavior of both species because larval stages are known to be consumed by M. nattereri (Hope et al., 2014). Alternatively, prey classified as not active nocturnal fliers can be captured by aerial hawking (e.g., ballooning spiders). More generally, arthropod nocturnal aerial activity is not straightforward to categorize, and therefore, our classification is only tentative. However, potential classification biases are expected to be low and standardized across species because the arthropod orders that are most difficult to categorize due their functional diversity, like Coleoptera, are consumed in similarly low proportion by both bat species. Nevertheless, due to this and low sample sizes in sympatric locations, interpretations of functional prey shift should be considered with caution.

\section{5 | CONCLUSIONS}

In line with niche theory predictions, we show that coexistence among morphologically identical (cryptic) species can be facilitated through fine-scale mechanisms of resource partitioning, even between species that show high levels of trophic similarity. Our findings that trophic resource partitioning is only detected when bats are syntopic within areas of sympatry suggest that fine-scale mechanisms of coexistence could have implications for the maintenance of broad-scale diversity patterns and highlight the importance of using appropriated spatial scales when studying impacts of biotic interactions on community assembly. This is the first study to identify a trophic shift between allotopic and syntopic populations of insectivorous bats, supporting the role of trophic resource partitioning in enabling species co-occurrence in the same foraging site. It thereby addresses some of the key limitations identified in a recent review 
of interspecific competition in bats (Salinas-Ramos et al., 2020). We highlight the importance of using high taxonomic resolution and allopatric populations at meaningful spatial scales for identifying patterns of niche shift, and the utility of using a functional approach that better links mechanistically with species trophic ecology. Understanding mechanisms of coexistence is essential for predicting species vulnerability under climate change because range shifts will result in new community assemblages and competitive interactions (HilleRisLambers et al., 2013). This is particularly relevant in our study system as both species are restricted to the Mediterranean region, where climate change is predicted to be particularly severe (Sala et al., 2000), and both are predicted to experience range shifts and changes in range overlap under climate change (Razgour et al., 2019).

\section{ACKNOWLEDGMENTS}

We are grateful to the following people who assisted with fieldwork: Raúl Molleda, Carmen Soria, Daniel Fernández, Pedro Alonso, Juan Tomás Alcalde, Xavier Puig and Maria Mas, Jesús Nogueras, and Sonia Sánchez. We also thank Jorge Mederos for his help in designing the arthropod sampling and functional classification, and Juan Luis García-Mudarra for his assistance with laboratory work and comments on the manuscript. Permits to carry out the fieldwork were provided by the administrations of Junta de Andalucía, Gobierno de La Rioja and Cantabria, and Junta de Castilla y León. RNF was funded through a School of Biological Science studentship at the University of Southampton. OR was funded through a Natural Environment Research Council Independent Research Fellowship (NE/M018660/1). Sampling in Jaén was funded by the MICINN project (CGL 2012-38610).

\section{AUTHOR CONTRIBUTION}

Roberto Novella-Fernandez: Data collection (lead); Formal analysis (lead); Investigation (lead); Methodology (equal); Resources (lead); Visualization (lead); Writing-original draft (lead). Carlos Ibáñez: Data curation (equal); Investigation (supporting); Resources (supporting); Supervision (supporting); Writing-review \& editing (equal). Javier Juste: Conceptualization (supporting); Data curation (equal); Investigation (supporting); Resources (equal); Supervision (supporting); Writing-review \& editing (equal). Elizabeth L. Clare: Formal analysis (supporting); Methodology (supporting); Writing-review \& editing (equal). C. Patrick Doncaster: Methodology (supporting); Supervision (supporting); Writing-review \& editing (equal). Orly Razgour: Conceptualization (lead); Funding acquisition (lead); Methodology (equal); Supervision (lead); Writing-review \& editing (equal).

\section{DATA AVAILABILITY STATEMENT}

Metabarcoding sequencing data are deposited in Dryad (https://doi. org/10.5061/dryad.c59zw3r5s). List of arthropod BINs identified in bat fecal samples and their presence in each of the two bat species has been uploaded as Data file S1.

\section{ORCID}

C. Patrick Doncaster iD https://orcid.org/0000-0001-9406-0693

Orly Razgour (iD https://orcid.org/0000-0003-3186-0313

\section{REFERENCES}

Agosta, S. J., Morton, D., \& Kuhn, K. M. (2003). Feeding ecology of the bat Eptesicus fuscus: "Preferred" prey abundance as one factor influencing prey selection and diet breadth. Journal of Zoology, 260, 169-177. https://doi.org/10.1017/S0952836903003601

Alberdi, A., Aizpurua, O., Thomas, M., Gilbert, P., \& Bohmann, K. (2018). Scrutinizing key steps for reliable metabarcoding of environmental samples (A Mahon, Ed.). Methods in Ecology and Evolution, 9, 134-147.

Albrecht, M., \& Gotelli, N. J. (2001). Spatial and temporal niche partitioning in grassland ants. Oecologia, 126, 134-141. https://doi. org/10.1007/s004420000494

Aldasoro, M., Garin, I., Vallejo, N., Baroja, U., Arrizabalaga-Escudero, A., Goiti, U., \& Aihartza, J. (2019). Gaining ecological insight on dietary allocation among horseshoe bats through molecular primer combination (B Schierwater, Ed.). PLoS One, 14, e0220081.

Alonso, D., Etienne, R., \& Mckane, A.(2006). The merits of neutral theory. Trends in Ecology \& Evolution, 21, 451-457. https://doi.org/10.1016/j. tree.2006.03.019

Andreas, M., Reiter, A., \& Benda, P. (2012a). Dietary composition, resource partitioning and trophic niche overlap in three forest foliage-gleaning bats in Central Europe. Acta Chiropterologica 14, 335-345.

Andreas, M., Reiter, A., \& Benda, P. (2012b). Prey selection and seasonal diet changes in the western barbastelle bat (Barbastella barbastellus). Acta Chiropterologica 14, 81-92.

Arlettaz, R. (1996). Foraging behaviour of the gleaning bat Myotis nattereri (Chiroptera, Vespertilionidae) in the Swiss Alps. Mammalia, 60, 181-186.

Arlettaz, R. (1999). Habitat selection as a major resource partitioning mechanism between the two sympatric sibling bat species Myotis myotis and Myotis blythii. Journal of Animal Ecology, 68, 460-471.

Arrizabalaga-Escudero, A., Clare, E. L., Salsamendi, E., Alberdi, A., Garin, I., Aihartza, J., \& Goiti, U. (2018). Assessing niche partitioning of co-occurring sibling bat species by DNA metabarcoding. Molecular Ecology, 27, 1273-1283. https://doi.org/10.1111/mec.14508

Ashrafi, S., Beck, A., Rutishauser, M., Arlettaz, R., \& Bontadina, F. (2011). Trophic niche partitioning of cryptic species of long-eared bats in Switzerland: Implications for conservation. European Journal of Wildlife Research, 57, 843-849. https://doi.org/10.1007/s1034 4-011-0496-z

Barclay, R. M. R. (1991). Population structure of temperate zone insectivorous bats in relation to foraging behaviour and energy demand. The Journal of Animal Ecology, 60, 165-178. https://doi.org/10.2307/5452

Bocher, P., Robin, F., Kojadinovic, J., Delaporte, P., Rousseau, P., Dupuy, C., \& Bustamante, P. (2014). Trophic resource partitioning within a shorebird community feeding on intertidal mudflat habitats. Journal of Sea Research, 92, 115-124. https://doi.org/10.1016/j. seares.2014.02.011

Böhm, S. M., Wells, K., \& Kalko, E. K. V. (2011). Top-down control of herbivory by birds and bats in the canopy of temperate broad-leaved oaks (Quercus robur). PLoS One, 6(4), e17857. in press. https://doi. org/10.1371/journal.pone.0017857

Boye, P., \& Dietz, M. (2005). Development of good practice guidelines for woodland management for bats. Report 661, English Nature Research Reports, Peterborough, UK: English Nature. http://publications.naturalengland.org.uk/publication/65012.

Chase, J. M., \& Leibold, M. A. (2003). Ecological Niches. University of Chicago Press. 
Chesson, P. (2000). Mechanisms of maintenance of species diversity. Annual Review of Ecology and Systematics, 31, 343-366. https://doi. org/10.1146/annurev.ecolsys.31.1.343

Chesson, P. L., \& Warner, R. R. (1981). Environmental variability promotes coexistence in lottery competitive systems. The American Naturalist, 117, 923-943. https://doi.org/10.1086/283778

Codron, J., Duffy, K. J., Avenant, N. L., Sponheimer, M., Leichliter, J., Paine, O., Sandberg, P., \& Codron, D. (2015). Stable isotope evidence for trophic niche partitioning in a South African savanna rodent community. Current Zoology, 61, 397-411. https://doi.org/10.1093/czool o/61.3.397

Cooper, R., \& Whitmore, R. C. (1990). Arthropod sampling methods in ornithology. Studies in Avian Biology, 13, 29-37.

Czech, N. U., Klauer, G., Dehnhardt, G., \& Siemers, B. M. (2009). Fringe for foraging? Histology of the bristle-like hairs on the tail membrane of the gleaning bat, Myotis nattereri. Acta Chiropterologica, 10, 303-311.

de Jong, J. (1995). Habitat use and species richness of bats in a patchy lanscape. Acta Theriologica (warsz). 40, 237-248.

Deagle, B. E., Thomas, A. C., Mclnnes, J. C., Clarke, L. J., Vesterinen, E. J., Clare, E. L., Kartzinel, T. R., \& Paige Eveson, J. (2019). Counting with DNA in metabarcoding studies: How should we convert sequence reads to dietary data? Molecular Ecology, 28, 391-406.

Edgar, R. C. (2010). Search and clustering orders of magnitude faster than BLAST. Bioinformatics, 26, 2460-2461. https://doi.org/10.1093/ bioinformatics/btq461

Elbrecht, V., Braukmann, T. W. A., Ivanova, N. V., Prosser, S. W. J., Hajibabaei, M., Wright, M., Zakharov, E. V., Hebert, P. D. N., \& Steinke, D. (2019). Validation of COI metabarcoding primers for terrestrial arthropods. PeerJ, 7, e7745. https://doi.org/10.7717/peerj.7745

Emrich, M. A., Clare, E. L., Symondson, W. O. C., Koenig, S. E., \& Fenton, M. B. (2014). Resource partitioning by insectivorous bats in Jamaica. Molecular Ecology, 23, 3648-3656. https://doi.org/10.1111/ mec.12504

Esnaola, A., Arrizabalaga-Escudero, A., González-Esteban, J., Elosegi, A., \& Aihartza, J. (2018). Determining diet from faeces: Selection of metabarcoding primers for the insectivore Pyrenean desman (Galemys pyrenaicus). PLoS One, 13, e0208986. https://doi.org/10.1371/journ al.pone.0208986

Gabaldón, C., Montero-Pau, J., Serra, M., \& Carmona, M. J. (2013). Morphological similarity and ecological overlap in two rotifer species. PLoS One, 8, 23-25. https://doi.org/10.1371/journal.pone.0057087

Galan, M., Pons, J.-B., Tournayre, O., Pierre, É., Leuchtmann, M., Pontier, D., \& Charbonnel, N. (2018). Metabarcoding for the parallel identification of several hundred predators and their prey: Application to bat species diet analysis. Molecular Ecology Resources, 18, 474-489. https://doi.org/10.1111/1755-0998.12749

Garcia-Mudarra, J. L. et al. (In Press). A PCR-based identification method of two bat cryptic complexes (Myotis crypticus/M. escalerai and Eptesicus isabellinus/E. serotinus). Journal of Bat Research Conservation. in press.

Gkenas, C., Magalhães, M. F., Cucherousset, J., Orjuela, R. L., \& Ribeiro, F. (2019). Dietary niche divergence between two invasive fish in Mediterranean streams. Knowledge \& Management of Aquatic Ecosystems, 420, 24 in press. https://doi.org/10.1051/kmae/2019018

Godsoe, W., Murray, R., \& Plank, M. (2015). The effect of competition on species' distributions depends on coexistence, rather than scale alone. Ecography (Cop.), 38, 1071-1079.

Gotelli, N., Hart, E. M., \& Ellison, A. M. (2015). EcoSimR: Null model analysis for ecological data, R package v 0.1.0. CRAN. https://github.com/ GotelliLab/EcoSimR/

Harmáčková, L., Remešová, E., \& Remeš, V. (2019). Specialization and niche overlap across spatial scales: Revealing ecological factors shaping species richness and coexistence in Australian songbirds (X Si, Ed.). Journal of Animal Ecology, 88, 1766-1776. https://doi. org/10.1111/1365-2656.13073
Hart, S. P., Usinowicz, J., \& Levine, J. M. (2017). The spatial scales of species coexistence. Nature Ecology \& Evolution, 1, 1066-1073. https:// doi.org/10.1038/s41559-017-0230-7

Harvey, J. A., Snaas, H., Malcicka, M., Visser, B., \& Bezemer, T. M. (2014). Small-scale spatial resource partitioning in a hyperparasitoid community. Arthropod-Plant Interactions, 8, 393-401. https://doi. org/10.1007/s11829-014-9319-y

HilleRisLambers, J., Harsch, M. A., Ettinger, A. K., Ford, K. R., \& Theobald, E. J. (2013). How will biotic interactions influence climate change-induced range shifts? Annals of the New York Academy of Sciences, 1297, 112-125. https://doi.org/10.1111/nyas.12182

Hope, P. R., Bohmann, K., Gilbert, M. T. P., Zepeda-Mendoza, M., Razgour, O., \& Jones, G. (2014). Second generation sequencing and morphological faecal analysis reveal unexpected foraging behaviour by Myotis nattereri (Chiroptera, Vespertilionidae) in winter. Frontiers in Zoology, 11, 39. https://doi.org/10.1186/1742-9994-11-39

Hubbell, S. P. (2001). The unified neutral theory of biodiversity and biogeography. Princeton University Press.

Huey, R. B., Pianka, E. R., Egan, M. E., \& Coons, L. W. (1974). Ecological shifts in sympatry: Kalahari fossorial lizards (Typhlosaurus). Ecology, 55, 304-316. https://doi.org/10.2307/1935218

Jiang, T., Feng, J., Sun, K., \& Wang, J. (2008). Coexistence of two sympatric and morphologically similar bat species Rhinolophus affinis and Rhinolophus pearsoni. Progress in Natural Science, 18, 523-532. https://doi.org/10.1016/j.pnsc.2007.12.005

Jones, G. (1990). Prey selection by the greater horseshoe bat (Rhinolophus ferrumequinum): Optimal foraging by echolocation?. Journal of Animal Ecology, 59, 587-602. https://doi.org/10.2307/4882

Jusino, M. A., Banik, M. T., Palmer, J. M., Wray, A. K., Xiao, L., Pelton, E., Barber, J. R., Kawahara, A. Y., Gratton, C., Peery, M. Z., \& Lindner, D. L.(2019). An improved method for utilizing high-throughput amplicon sequencing to determine the diets of insectivorous animals. Molecular Ecology Resources, 19, 176-190. https://doi. org/10.1111/1755-0998.12951

Juste, J., Ruedi, M., Puechmaille, S. J., Salicini, I., \& Ibáñez, C. (2019). Two new cryptic bat species within the Myotis nattereri species complex (Vespertilionidae, Chiroptera) from the western Palaearctic. Acta Chiropterologica, 20, 285.

Kalka, M. B., Smith, A. R., \& Kalko, E. K. V. (2008). Bats limit arthropods and herbivory in a tropical forest. Science (80-., ). 320, 71. https://doi. org/10.1126/science.1153352

Klawinski, P.D., Vaughan, R. K.,Saenz, D., \& Godwin, W. (1994).Comparison of dietary overlap between allopatric and sympatric geckos. Journal of Herpetology, 28, 225. https://doi.org/10.2307/1564624

Krüger, F., Clare, E. L., Greif, S., Siemers, B. M., Symondson, W. O. C., \& Sommer, R. S. (2014). An integrative approach to detect subtle trophic niche differentiation in the sympatric trawling bat species Myotis dasycneme and Myotis daubentonii. Molecular Ecology, 23, 3657-3671.

Kunz, T. H. (1973). Resource utilization: Temporal and spatial components of bat activity in central lowa. Journal of Mammalogy, 54, 1432. https://doi.org/10.2307/1378869

Kunz, T. H. (2009). Ecological and behavioral methods for the study of bats (TH Kunz, Ed.). Johns Hopkins University Press.

Lamarre, G. P. A., Hérault, B., Fine, P. V. A., Vedel, V., Lupoli, R., Mesones, I., \& Baraloto, C. (2016). Taxonomic and functional composition of arthropod assemblages across contrasting Amazonian forests. Journal of Animal Ecology, 85, 227-239. https://doi. org/10.1111/1365-2656.12445

Lawlor, L. R. (1980). Overlap, similarity, and competition coefficients. Ecology, 61, 245-251.

Letten, A. D., Ke, P.-J., \& Fukami, T. (2017). Linking modern coexistence theory and contemporary niche theory. Ecological Monographs, 87, 161-177. https://doi.org/10.1002/ecm.1242

Lewis, J. S., Bailey, L. L., VandeWoude, S., \& Crooks, K. R. (2015). Interspecific interactions between wild felids vary across scales and 
levels of urbanization. Ecology and Evolution, 5, 5946-5961. https:// doi.org/10.1002/ece3.1812

Luiselli, L. (2008). Do lizard communities partition the trophic niche? A worldwide meta-analysis using null models. Oikos, 117, 321-330. https://doi.org/10.1111/j.2007.0030-1299.16297.x

Martin, M. (2011). Cutadapt removes adapter sequences from high-throughput sequencing reads. EMBnet.journal, 17, 10. https:// doi.org/10.14806/ej.17.1.200

McGill, B. J., Maurer, B. A., \& Weiser, M. D. (2006). Empirical evaluation of neutral theory. Ecology, 87, 1411-1423. 10.1890/0012-9658(2006)87[1411:EEONT]2.0.CO;2

Norberg, U. M. (1994). Wing design, flight performance, and habitat use in bats. In P. C. Wainwright, \& S. M. Reilly (Eds.), Ecological morphology: Integrative organismal biology (p. 367). University of Chicago Press.

Oksanen, J. et al (2019). vegan: Community ecology package. R package v2.5-6, CRAN. https://github.com/vegandevs/vegan/

Peixoto, F. P., Braga, P. H. P., \& Mendes, P. (2018). A synthesis of ecological and evolutionary determinants of bat diversity across spatial scales. BMC Ecology, 18, 1-14. https://doi.org/10.1186/s1289 8-018-0174-z

R core team (2020). R: A language and environment for statistical computing. $\mathrm{R}$ core team in press.

Ratnasingham, S., \& Hebert, P. D. N. (2007). BARCODING: Bold: The Barcode of Life data system (http://www.barcodinglife.org). Molecular Ecology Notes, 7, 355-364. https://doi. org/10.1111/j.1471-8286.2007.01678.x

Ratnasingham, S., \& Hebert, P. D. N. (2013). A DNA-based registry for all animal species: The Barcode Index Number (BIN) system (D Fontaneto, Ed.). PLoS One, 8, e66213. https://doi.org/10.1371/journ al.pone.0066213

Razgour, O., Clare, E. L., Zeale, M. R. K., Hanmer, J., Schnell, I. B., Rasmussen, M., Gilbert, T. P., \& Jones, G. (2011). High-throughput sequencing offers insight into mechanisms of resource partitioning in cryptic bat species. Ecology and Evolution 1, 556-570. https://doi. org/10.1002/ece3.49

Razgour, O., Forester, B., Taggart, J. B., Bekaert, M., Juste, J., Ibáñez, C., Puechmaille, S. J., Novella-Fernandez, R., Alberdi, A., \& Manel, S. (2019). Considering adaptive genetic variation in climate change vulnerability assessment reduces species range loss projections. Proceedings of the National Academy of Sciences, 116, 10418-10423. https://doi.org/10.1073/pnas.1820663116

Razgour, O., Salicini, I., Ibáñez, C., Randi, E., \& Juste, J. (2015). Unravelling the evolutionary history and future prospects of endemic species restricted to former glacial refugia. Molecular Ecology, 24, 5267-5283. https://doi.org/10.1111/mec.13379

Rivers, N. M., Butlin, R. K., \& Altringham, J. D. (2005). Genetic population structure of Natterer's bats explained by mating at swarming sites and philopatry. Molecular Ecology, 14, 4299-4312. https://doi. org/10.1111/j.1365-294X.2005.02748.x

Ross, S. T. (1986). Resource partitioning in fish assemblages: Review of field studies. Copeia, 2, 352-388.

Russo, D., Di Febbraro, M., Rebelo, H., Mucedda, M., Cistrone, L., Agnelli, P., Pasquale, P. P. D., Martinoli, A., Scaravelli, D., Spilinga, C., \& Bosso, L. (2014). What story does geographic separation of insular bats tell? A case study on Sardinian rhinolophids (RM Brigham, Ed.). PLoS One, 9, e110894.

Rytkönen, S., Vesterinen, E. J., Westerduin, C., Leviäkangas, T., Vatka, E., Mutanen, M., Välimäki, P., Hukkanen, M., Suokas, M., \& Orell, M. (2019). From feces to data: A metabarcoding method for analyzing consumed and available prey in a bird-insect food web. Ecology and Evolution, 9, 631-639. https://doi.org/10.1002/ece3.4787

Sala, O. E., Chapin, F. S. 3rd, Armesto, J. J., Berlow, E., Bloomfield, J., Dirzo, R., Huber-Sanwald, E., Huenneke, L. F., Jackson, R. B., Kinzig, A., Leemans, R., Lodge, D. M., Mooney, H. A., Oesterheld, M., Poff,
N. L., Sykes, M. T., Walker, B. H., Walker, M., \& Wall, D. H. (2000). Global biodiversity scenarios for the year 2100. Science (80-. ). 287, 1770-1774.

Salinas-Ramos, V. B., Ancillotto, L., Bosso, L., Sánchez-Cordero, V., \& Russo, D. (2020). Interspecific competition in bats: State of knowledge and research challenges. Mammal Review, 50, 68-81. https:// doi.org/10.1111/mam.12180

Salsamendi, E., Garin, I., Almenar, D., Goiti, U., Napal, M., \& Aihartza, J. (2008). Diet and prey selection in Mehelyi's horseshoe bat Rhinolophus mehelyi (Chiroptera, Rhinolophidae) in the south-western Iberian Peninsula. Acta Chiropterologica, 10, 279-286.

Salsamendi, E., Garin, I., Arostegui, I., Goiti, U., \& Aihartza, J. (2012). What mechanism of niche segregation allows the coexistence of sympatric sibling rhinolophid bats? Frontiers in Zoology, 9, 30. https:// doi.org/10.1186/1742-9994-9-30

Schmitt, R. J., \& Coyer, J. A. (1983). Variation in surfperch diets between allopatry and sympatry: Circumstantial evidence for competition. Oecologia, 58, 402-410. https://doi.org/10.1007/BF00385243

Schoener, T. W. (1974). Resource partitioning in ecological communities. Science (80-.). 185, 27-39. https://doi.org/10.1126/scien ce.185.4145.27

Shiel, C. B., McAney, C. M., \& Fairley, J. S. (1991). Analysis of the diet of Natterer's bat Myotis nattereri and the common long-eared bat Plecotus auritus in the West of Ireland. Journal of Zoology, 223, 299-305.

Snyder, R. E., \& Chesson, P. (2004). How the spatial scales of dispersal, competition, and environmental heterogeneity interact to affect coexistence. American Naturalist, 164, 633-650.

Sousa, L. L., Silva, S. M., \& Xavier, R. (2019). DNA metabarcoding in diet studies: Unveiling ecological aspects in aquatic and terrestrial ecosystems. Environmental DNA, 1, 199-214. https://doi.org/10.1002/ edn3.27

Swift, S. M. (1997). Roosting and foraging behaviour of Natterer's bats (Myotis nattereri) close to the northern border of their distribution. Journal of Zoology, 242, 375-384. https://doi. org/10.1111/j.1469-7998.1997.tb05809.x

Swift, S. M., \& Racey, P. A. (2002). Gleaning as a foraging strategy in Natterer's bat Myotis nattereri. Behavioral Ecology and Sociobiology, 52, 408-416. https://doi.org/10.1007/s00265-002-0531-x

Ulyshen, M. D. (2011). Arthropod vertical stratification in temperate deciduous forests: Implications for conservation-oriented management. Forest Ecology and Management, 261, 1479-1489. https://doi. org/10.1016/j.foreco.2011.01.033

Valladares, F., Bastias, C. C., Godoy, O., Granda, E., \& Escudero, A. (2015). Species coexistence in a changing world. Frontiers in Plant Science, 6, 1-16. https://doi.org/10.3389/fpls.2015.00866

Vallejo, N., Aihartza, J., Goiti, U., Arrizabalaga-Escudero, A., Flaquer, C., Puig, X., Aldasoro, M., Baroja, U., \& Garin, I. (2019). The diet of the notch-eared bat (Myotis emarginatus) across the Iberian Peninsula analysed by amplicon metabarcoding. Hystrix, 30, 59-64.

Vaughan, N. (1997). The diets of British bats (Chiroptera). Mammal Review, 27, 77-94. https://doi.org/10.1111/j.1365-2907.1997.tb003 73.x

Vesterinen, E. J., Ruokolainen, L., Wahlberg, N., Peña, C., Roslin, T., Laine, V. N., Vasko, V., Sääksjärvi, I. E., Norrdahl, K., \& Lilley, T. M. (2016). What you need is what you eat? Prey selection by the bat Myotis daubentonii. Molecular Ecology, 25, 1581-1594.

Viana, D. S., \& Chase, J. M. (2019). Spatial scale modulates the inference of metacommunity assembly processes. Ecology, 100, 1-9. https:// doi.org/10.1002/ecy.2576

Wainwright, P., \& Reilly, S. (1994). Ecological morphology: Integrative organismal biology. University of Chicago Press.

Weiher, E., \& Keddy, P. (1999) Ecological assembly rules, perspectives, advances, retreats (E Weiher and P Keddy, Eds.). Cambridge University Press. 
Zeale, M. R. K., Butlin, R. K., Barker, G. L. A., Lees, D. C., \& Jones, G. (2011). Taxon-specific PCR for DNA barcoding arthropod prey in bat faeces. Molecular Ecology Resources, 11, 236-244. https://doi. org/10.1111/j.1755-0998.2010.02920.x

\section{SUPPORTING INFORMATION}

Additional supporting information may be found online in the Supporting Information section.
How to cite this article: Novella-Fernandez R, Ibañez C, Juste J, Clare EL, Doncaster CP, Razgour O. Trophic resource partitioning drives fine-scale coexistence in cryptic bat species. Ecol Evol. 2020;10:14122-14136. https://doi.org/10.1002/ ece3.7004 